\title{
Comparison of Cf-252 Thin-Film Sources Prepared by Evaporation or Self-Transfer
}

\author{
N. J. Algutifan ${ }^{1}$, S. R. Sherman ${ }^{2}$, and C. W. Alexander \\ Nuclear Materials Processing Group, Nuclear Security and Isotope Technology Division, Oak Ridge \\ National Laboratory, 1 Bethel Valley Road, Oak Ridge, TN 37831
}

\begin{abstract}
Thin-film sources containing Cf-252 were prepared by two techniques-evaporation and self-transferto determine whether sources prepared by simple evaporation work as well as sources prepared by selftransfer for alpha particle studies. The sources were analyzed by alpha and gamma spectroscopy.

Results indicate that self-transfer sources exhibit less alpha energy straggling and alpha energy loss than evaporative sources. Fission fragments may also self-transfer, and sources made by self-transfer may need time to decay before reaching radioactive equilibrium.
\end{abstract}

Notice: This manuscript has been authored by UT-Battelle, LLC, under Contract No. DE-AC05-000R22725 with the U.S. Department of Energy. The United States Government retains and the publisher, by accepting the article for publication, acknowledges that the United States Government retains a non-exclusive, paid-up, irrevocable, worldwide license to publish or reproduce the published form of this manuscript, or allow others to do so, for United States Government purposes.

\footnotetext{
${ }^{1}$ Deceased June 2013.

${ }^{2}$ Corresponding author, Phone: (865) 576-8267, E-mail: shermansr@ornl.gov
} 


\section{INTRODUCTION}

Californium-252 ( $\mathrm{Cf}-252, \mathrm{Z}=98$ ) is a potent neutron generator due to its propensity to decay by spontaneous fission. Approximately 3.09\% of Cf-252 atoms fission into fragments upon decay, releasing on average 3.77 neutrons per fission event. The balance of atoms decay by alpha emission to form Curium-248 $(\mathrm{Cm}-248, \mathrm{Z}=96)$. Californium-252 has a 2.645 year half-life and emits $2.3 \times 10^{6}$ neutrons per microgram per second due to spontaneous fission (Haire, 2011). As a neutron generator, Cf-252 has found wide use in sealed-source form for prompt-gamma neutron activation analysis, nuclear reactor start-up, neutron radiography, and other uses (Osborne-Lee and Alexander, 1995).

Sealed sources containing Cf-252 are very useful tools, but they are less useful for scientific study of Cf-252 because the encapsulating material blocks detection of the alpha particles and fission decay fragments. An exposed Cf-252 source is needed for such studies.

A thin-film exposed source is better yet. A thin-film source is defined as a source that is sufficiently thin that the source material does not significantly interfere with its own particle emissions outside the plane of the source. Penetrating gamma and neutron emissions are generally unaffected by the thickness of a thin-film source, but the track lengths of alpha particles and fission fragments are very short in solids, and absolute particle counts and measurements of particle energies are adversely affected by source self-shielding. For those types of particle studies, a thin-film radioactive source must be sufficiently thin that self-shielding has little effect on the particle emission measurements.

This work was undertaken to determine whether thin-film Cf-252 sources prepared by simple evaporation are comparable to thin-film sources prepared by self-transfer in regard to alpha particle measurements. Evaporative sources are prepared by dripping a solution containing Cf-252 onto a solid surface and heating the surface until the droplet dries to form a solid residue. Self-transfer sources are prepared in a vacuum and rely on the spontaneous transfer or self-sputtering of Cf- 252 from an exposed 
mother source to a receiver plate placed close to it (Pauker and Steiger-Shafrir, 1971). The self-transfer method is known to produce thin films, but the evaporation method is easier to perform and requires less source material. Data are presented to show the similarities and differences between sources prepared by these methods. The self-transfer sources exhibit less alpha energy straggling than the evaporative sources, but they also emit more gamma rays, which are attributed to the fission products that transferred along with the Cf-252. Work is ongoing to determine whether and when the selftransfer sources reach radioactive equilibrium, a state in which the rate of radioactive decay of the source is driven by the decay rate of Cf- 252 .

\section{EXPERIMENTS}

\subsection{Instrumentation}

Alpha spectroscopy measurements were made using a Canberra alpha spectrometer (Model 7401) with a Canberra passivated implanted planar silicon detector. Gamma spectroscopy measurements were made using a Canberra standard-electrode coaxial germanium detector (Model GC6020). The micrometer used to measure source areas was precise to within $\pm 0.0025 \mathrm{~cm}$. A Canberra Vacuum Chamber (Model 7400A) was used to make the self-transfer sources.

\subsection{Evaporative Source Preparation and Analysis}

A $1 \mathrm{~mL}$ liquid sample containing $2.89 \times 10^{4} \mathrm{~Bq} \mathrm{Cf}$ in $0.1 \mathrm{M} \mathrm{HNO}_{3}$ was obtained from the Radiochemical Engineering Development Center at Oak Ridge National Laboratory (ORNL) in Oak Ridge, Tennessee, United States. The isotopic composition (atom\%) of the Cf in the liquid sample was: Cf-249, 12.68\%; Cf-250, 15.27\%; Cf-251, 5.53\%; Cf-252, 66.52\%; Cm isotopes, $0 \%$.

Three evaporative sources were prepared from the liquid Cf sample. Source \#1 was made by evaporating $50 \mu \mathrm{L}$ of the solution onto a small flat stainless steel disk. The disk was placed onto a hot plate and heated to $85^{\circ} \mathrm{C}$ in a radiological fume hood. Then, droplets of the liquid were dripped onto the 
plate using a volumetric pipet until $50 \mu \mathrm{L}$ had been dispensed. The droplets were allowed to dry between applications. Sources \#2 and \#3 were prepared similarly; with the exception that $100 \mu \mathrm{L}$ of the solution was used for Source \#2 and $125 \mu \mathrm{L}$ was used for Source \#3.

The sources were first analyzed by gamma spectroscopy. Then, the sources were heated briefly in a vacuum furnace to remove residual moisture. After drying, the sources were measured using alpha spectroscopy. The measured gamma and alpha spectra were compared against spectral data available in GENIE (General Internet Search Engine for Atomic Data), an online tool constructed and maintained by the International Atomic Energy Agency. PeakEasy, a spectral analysis software package from Los Alamos National Laboratory, was used to analyze the spectral data. Its Gaussian fitting algorithm was used to obtain region-of-interest (ROI) parameters, including total peak counts and peak full-width at half maximum (FWHM) energies. Output data from PeakEasy were imported into Microsoft Excel for graphing and analysis.

Source areas were calculated by measuring the length and width of the visible source residue using a micrometer and then averaging the two numbers to get an effective diameter. The effective source area was then calculated as the area of a circle.

\subsection{Self-Transfer Source Preparation and Analysis}

A flat, electrodeposited Cf-252 source having a diameter of $2.16 \mathrm{~cm}$ was used as the mother source. The isotopic composition (atom\%) of the mother source was: Cf-249, 2.47\%; Cf-250, 6.78\%; Cf251, 3.71\%; Cf-252, 5.87\%; Cm-245, 0.047\%; Cm-246, 4.81\%; Cm-247, 0\%; and Cm-248, 76.31\%.

Two sources were prepared by self-transfer. To prepare each source, the mother source was placed in the bottom of the vacuum chamber. Three metal washers were stacked on top of the source with their holes in alignment. The receiver plate, a $1.245 \mathrm{~cm}$-diameter platinum disk, was placed on top of the washers. The washers created a separation distance of about $75 \mu \mathrm{m}$ between the mother source and the receiver plate. After placement of the receiver plate, the vacuum chamber was sealed and 
pumped down to the lowest pressure that could be achieved, which was about $160 \mathrm{~mm} \mathrm{Hg}$ ( $0.2 \mathrm{~atm}$ ), and the chamber was held at that pressure for a number of hours. Source \#4 was exposed for $24 \mathrm{~h}$; Source \#5 was exposed for $67 \mathrm{~h}$.

Following exposure, the receiver plates were measured by gamma and alpha spectroscopy, and the spectra were analyzed using GENIE and PeakEasy in the same manner as the spectra for the evaporative sources.

The source areas were calculated using a micrometer to measure the diameter of the hole in the metal washers that were used to separate the mother source from the platinum receiver plate. The physical boundary of the thin-film deposit was not visible on the surface of the platinum, so it was assumed that the area of the self-transfer source was determined by the diameter of the hole in the washers, which acted as a collimator for the self-transferred material.

\section{RESULTS AND DISCUSSION}

The alpha spectra for the evaporative sources are shown in Figure 1, and the alpha spectra for the self-transfer sources are shown in Figure 2. Figure 3 shows an overlay of the alpha spectra for Sources \#1 (evaporation) and \#5 (self-transfer). The data were normalized by dividing the number of counts per channel by the total number of counts for each sample, and the energy range of interest was narrowed to show just the californium peaks.

The gamma spectra were measured for all of the sources. Figure 4 shows a comparison of the gamma spectra for Sources \#3 (evaporation) and \#5 (self-transfer). The peaks corresponding to Cf-249 and some of the more commonly produced gamma-emitting fission fragments (Skovorodkin et al., 1973) are designated on the plot. No Cf-251 peak was detected, and no labels were applied for Cf- 250 or Cf252 because these isotopes have broad undifferentiated gamma ray emissions due to spontaneous fissioning (Billnert et al., 2013). 
Table 1 shows the measured activities, linear densities, energy resolutions, and loss factors for the sources. These factors were calculated to characterize the alpha spectrum for each source. Linear density, $\rho_{L}$, was calculated by measuring the alpha activity of each source, converting the activity into a source mass, and dividing the source mass by the surface area of each source. The source surface areas were determined using the methods described in Sections 2.2 or 2.3. The energy resolution, $E R$, of each source was determined by identifying a ROI in the alpha spectra, in this case the $6.118 \mathrm{MeV}$ alpha peak for Cf-252 (Haire, 2011; Akovali, 1999), and then taking the ratio of the FWHM of the measured energy peak to the known peak's maximum energy. The loss factor, $L$, was calculated as the ratio of the theoretical alpha particle energy to the measured alpha particle energy for the ROI.

The evaporative sources show a leftward shift in the $6.118 \mathrm{MeV}$ alpha energy peak compared to the self-transfer sources, as shown in Figure 3. This leftward shift, called "alpha energy straggling" (Comfort et al., 1966), is presumably due to the greater thickness or surface density of the evaporative sources. There is no clear trend with the calculation of energy resolutions, and the $E R$ of Sources \#1 and \#5 are statistically the same. The loss factor calculations do show a difference, and the sources prepared by evaporation show a greater energy loss $(L>1.0)$ than those prepared by self-transfer $(L \approx 1.0)$.

The gamma spectra of the self-transfer sources showed a greater number of peaks that could be differentiated from background compared to the gamma spectra of the evaporative sources. A possible explanation for this difference may be that radioactive fission fragments also self-transported to the receiving plate, and the receiving plate was measured before it reached radioactive equilibrium. This would cause the relative concentration of fission products on the receiving plate to be higher than in the mother source or on a comparably sized evaporative source. Future work will include gamma spectroscopy measurements made over time to determine when a self-transfer source reaches radioactive equilibrium. 


\section{CONCLUSIONS}

The differences between sources prepared by the two methods - evaporation and selftransfer-are discernable. The sources prepared by self-transfer exhibit less alpha energy straggling and alpha energy loss than sources prepared by evaporation. The sources prepared by self-transfer showed additional peaks in the gamma spectra, indicating the transfer of shorter-lived fission fragments to the receiver plate. The activities of the sources prepared by self-transfer were considerably weaker, however, and required more preparation time and equipment. Because the self-transfer method allows for fabrication of thinner sources, it is a better method than evaporation for preparing sources for studying the alpha-particle and fission-fragment emissions from Cf-252 and other self-fissioning radioisotopes.

\section{ACKNOWLEDGMENTS}

The U.S. National Nuclear Security Administration (NNSA) Office of Nuclear Materials Integration (NA-

73) sponsored this work. This manuscript has been authored by UT-Battelle, LLC, under Contract No. DEAC05-00OR22725 with the U.S. Department of Energy. The authors thank Dr. Rose Boll (Oak Ridge National Laboratory) for providing the Cf-252 solution and mother source for this work.

\section{REFERENCES}

Akovali, Y. A., 1999. Nuclear Data Sheets for A = 248, 252, 256, 260, 264. Nuclear Data Sheets, 87, pp. 249-316.

Billnert, R., Hambsch, F.-J., Oberstedt, A., Oberstedt, S, 2013. New prompt spectral $\gamma$-ray data from the reaction ${ }^{252} \mathrm{Cf}(\mathrm{sf})$ and its implication on present evaluated nuclear data files. Physical Review C, 87, pp. 024601-1 to 024601-5, doi:10/1103/PhysRevC.87l.024601. 
Comfort, J. R., Decker, J. F., Lynk, E. T., Scully, M. O., Quinton, A. R., 1966. Energy Loss and Straggling of Alpha Particles in Metal Foils. Physical Review, 150, 1, pp. 249-256.

Haire, R. G., 2011. Californium, in: Morss, L. R., Edelstein, N. M., Fuger, J., Katz, J. J. (Eds.). The Chemistry of the Actinide and Transactinide Elements ( $4^{\text {th }}$ ed.), Vol. 3. Springer, Dordrecht, The Netherlands, pp. 1499-1576.

Osborne-Lee, I. W., Alexander, C. W., 1995. Californium-252: A Remarkable Versatile Isotope. ORNL/TM12706, Oak Ridge National Laboratory.

Pauker, S., Steiger-Shafrir, N. H., 1971. Transfer Properties of ${ }^{252} \mathrm{Cf}$ and their Use for Source Preparation. Nuclear Instruments and Methods, 91, pp. 557-563.

Skovorodkin, N. V., Lozhkomoev, G. E., Petrzhak, K. A., Sorokina, A. V., Aleksandrov, B. M., Krivokhatskii, A. S., 1973. Yields of Fragments of the Spontaneous Fission of $\mathrm{Cf}^{252}$. Atomnaya Energiya, 34, 5, pp. $365-371$.

Figure captions:

Figure 1. Alpha spectra for Evaporative Sources \#1 (घ), \#2 (४ ), and \#3 (•).

Figure 2. Alpha spectra for Self-Transfer Sources \#4 (घ) and \#5 ( $)$.

Figure 3. Overlay of alpha spectra for Sources \#1 (४) and \#5 (ロ).

Figure 4. Gamma spectra for Source \#3 (top curve) and Source \#5 (bottom curve). 
Table 1. Source Characteristics

\begin{tabular}{|c|c|c|c|c|}
\hline Source \# & Cf-252 Activity (Bq) & $\begin{array}{l}\text { Calculated Linear } \\
\text { Density } \\
\text { (picograms } / \mathrm{cm}^{2} \text { ) }\end{array}$ & $E R$ & $L$ \\
\hline \multicolumn{5}{|c|}{ Evaporation } \\
\hline 1 & $1,428 \pm 0.4$ & $33.60 \pm 0.01$ & 0.613 & 1.0023 \\
\hline 2 & $5,661 \pm 29$ & $56.30 \pm 0.28$ & 0.743 & 1.0033 \\
\hline 3 & $6,669 \pm 1$ & $736.00 \pm 0.11$ & 0.670 & 1.0044 \\
\hline \multicolumn{5}{|c|}{ Self-Transfer } \\
\hline 4 & $76 \pm 0.7$ & $3.30 \pm 0.03$ & 0.600 & 0.9990 \\
\hline 5 & $110 \pm 0.7$ & $4.80 \pm 0.004$ & 0.614 & 1.0000 \\
\hline
\end{tabular}

\section{Highlights}

- Thin film Cf-252 sources were prepared by evaporation and self-transfer.

- Sources were analyzed by alpha and gamma spectroscopy.

- Self-transfer sources have less alpha straggling and energy loss.

- Fission fragments may also self-transport. 


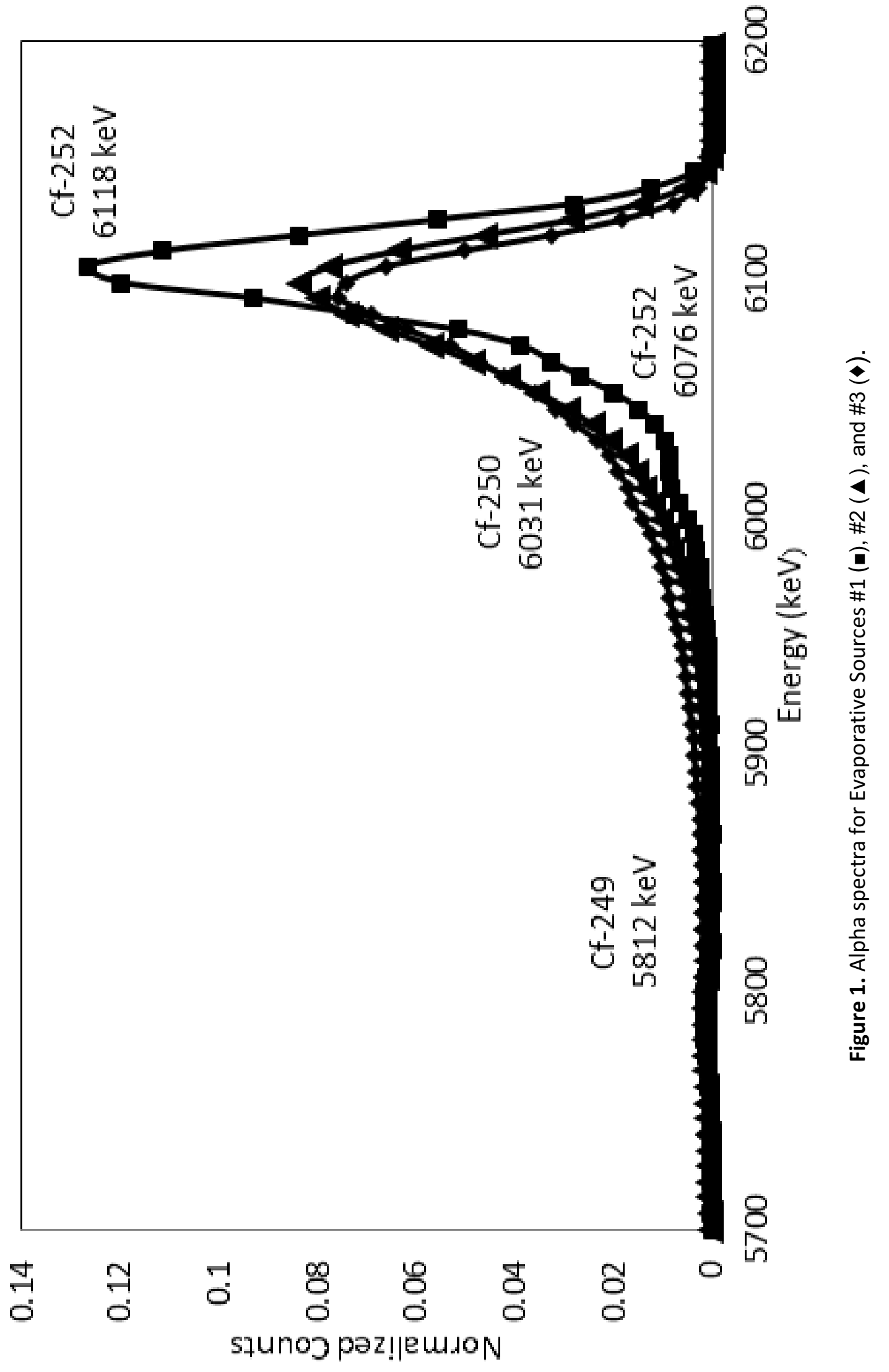




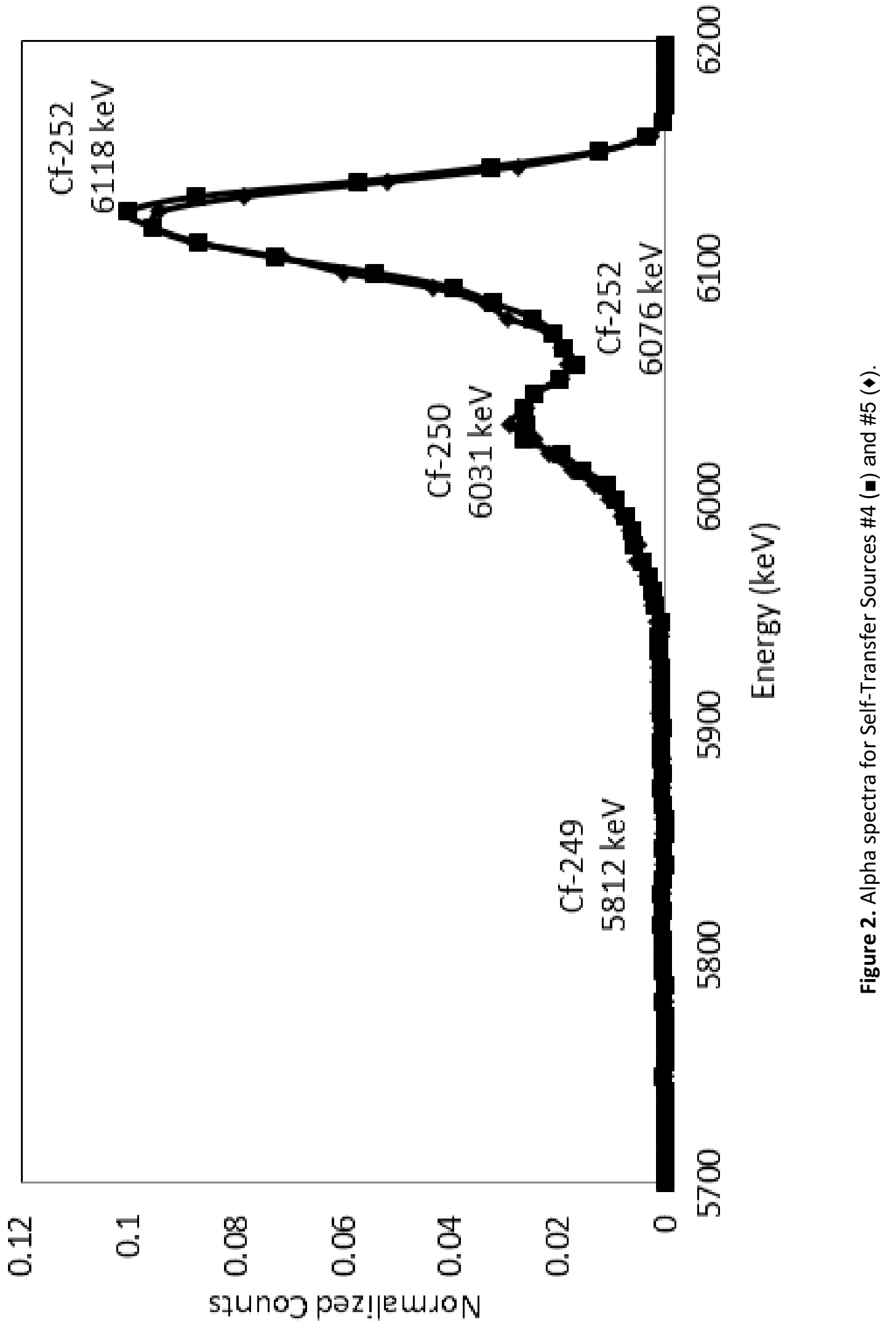




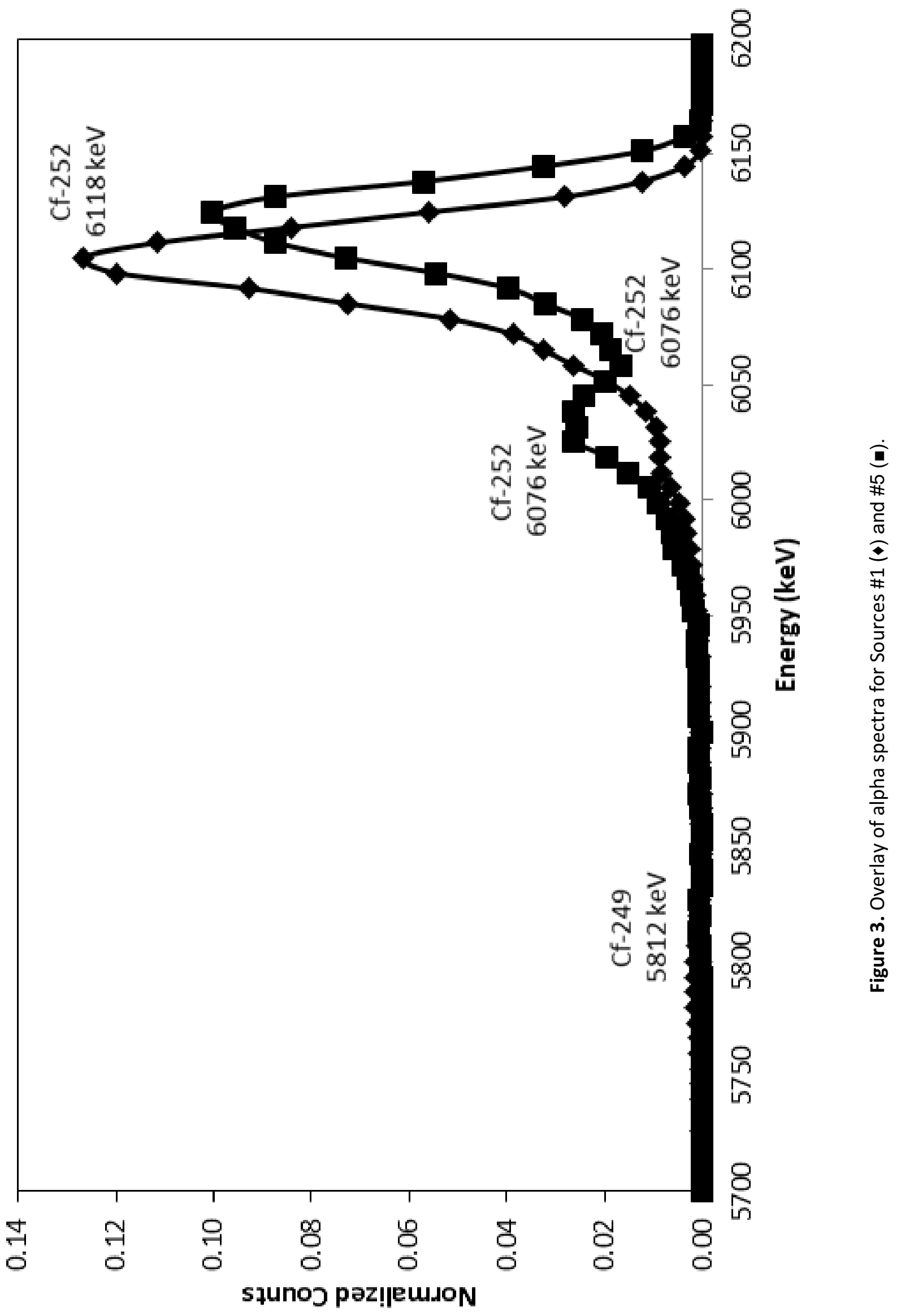




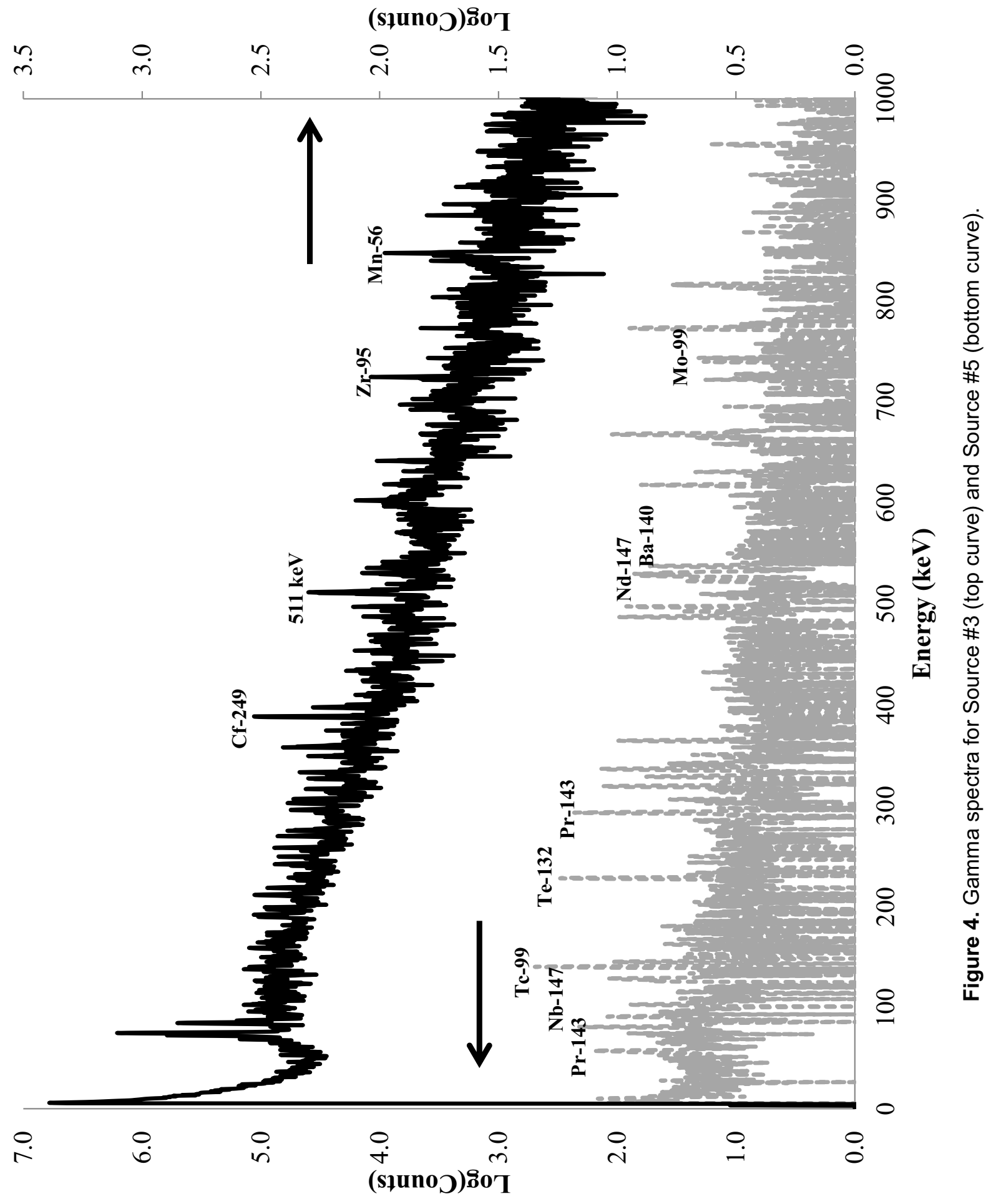

